Snaking of multiple homoclinic orbits in reversible systems

Knobloch, J. and Wagenknecht, T.

2007

MIMS EPrint: 2007.102

Manchester Institute for Mathematical Sciences

School of Mathematics

The University of Manchester

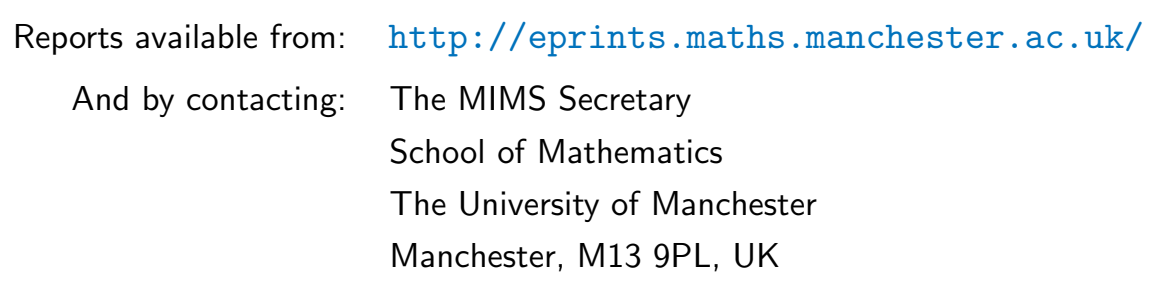

ISSN 1749-9097 


\title{
Snaking of multiple homoclinic orbits in reversible systems
}

\author{
J. Knobloch \\ Department of Mathematics, TU-Ilmenau, D-98684 Ilmenau, Germany \\ E-Mail: juergen.knobloch@tu-ilmenau.de
}

T. Wagenknecht ${ }^{1}$

School of Mathematics, University of Manchester, Sackville Street, Manchester, M60 1QD, UK

E-Mail: thomas.wagenknecht@manchester.ac.uk

\begin{abstract}
We study $N$-homoclinic orbits near a heteroclinic cycle in a reversible system. The cycle is assumed to connect two equilibria of saddle-focus type. Using Lin's method we establish the existence of infinitely many $N$-homoclinic orbits for each $N$ near the cycle. In particular, these orbits exist along snaking curves, thus mirroring the behaviour one-homoclinic orbits. The general analysis is illustrated by numerical studies for a Swift-Hohenberg system.
\end{abstract}

Key words: bifurcation, multiple homoclinic orbits, heteroclinic cycle, homoclinic snaking, Lin's method

PACS: $02.30 . \mathrm{Hq}, 05.45-\mathrm{a}$

\section{Introduction}

Spatially localised structures, such as solitary pulses, appear in many systems described by higher-order nonlinear partial differential equations (PDEs). Particular examples have been found in structural mechanics [7], nonlinear optics [15] and water wave problems [2]. A common feature of these cases is the onset of the localised patterns in a sequence of fold bifurcations, which are connected by a snaking curve.

$\overline{1}$ Corresponding author 
In one spatial dimension this phenomenon can be explained by a sequence of bifurcations in the associated ordinary differential equation (ODE) for travelling waves. Localised patterns correspond to homoclinic solutions of this ODE, and it has been found that infinitely many of such orbits can exist near a heteroclinic cycle in the ODE. These homoclinic orbits all lie on a snaking curve, along which they undergo infinitely many fold bifurcations, while thereby getting wider and developing new oscillations about their centre at each fold. An important requirement for this scenario to happen is the time-reversibility of the travelling-wave ODE.

In addition to time-reversibility the type of the heteroclinic cycle plays an important role for the dynamics. Homoclinic snaking has been observed in a neighbourhood of a heteroclinic cycle between an equilibrium $p_{1}$ and a saddle focus equilibrium $p_{2}$ (EE cycle) and near cycles connecting an equilibrium $p_{1}$ and a periodic orbit $P$ (EP cycle). The homoclinic orbits occurring along a snaking curve are asymptotic to $p_{1}$.

In the case of an EE cycle the snaking occurs locally around some critical value of a family parameter at which a codimension one heteroclinic cycle exists. This feature allows one to study the scenario using a local bifurcation analysis. In an earlier paper [10] it has been shown rigorously by the authors that heteroclinic cycles between symmetric equilibria of saddle focus type generate a snaking behaviour. In contrast to that behaviour the snaking related to an EP cycle is generically a global phenomenon. For a geometric explanation for why homoclinic snaking occurs in this case we refer to $[18,3]$.

If also $p_{1}$ is of saddle focus type, then general results by Häerterich [4], see also [14], show that homoclinic orbits to $p_{1}$ will be accompanied by a plethora of $N$-homoclinic orbits, i.e. homoclinic orbits to $p_{1}$ that pass $p_{2} N$ times before closing the loop. For each $N$ there exist infinitely many $N$-homoclinic orbits, which are distinguished by the times they spend near $p_{1}$. In the case studied here, one may now expect these orbits to snake under variation of the parameter, too. Our goal here is to describe this snaking.

More precisely, we understand a snaking curve as a graph $\{(\omega, \lambda(\omega)), \omega \in I\}$ that intersects a line $\left\{\left(\omega, \lambda^{*}\right), \omega \in I\right\}$ infinitely many times. Moreover, in the case of an EE cycle $\lambda(\omega)$ tends to $\lambda^{*}$ as $\omega$ tends to infinity. So, a snaking curve looks qualitatively similar to the one in Figure 1. Here $\omega$ is some intrinsic parameter characterising the the $N$-homoclinic orbit, taken from some infinite interval $I$, and $\lambda(\omega)$ is the family parameter of the ODE at which the $N$ homoclinic orbit exists. Roughly speaking, $\omega$ is the length of stay near $p_{2}$ during a certain passage of the $N$-homoclinic orbit near $p_{2}$. In the analysis in Section 4 , we will particularly focus on the case where $\omega$ is the first passage past $p_{2}$, and we will discuss other possibilities only briefly. 


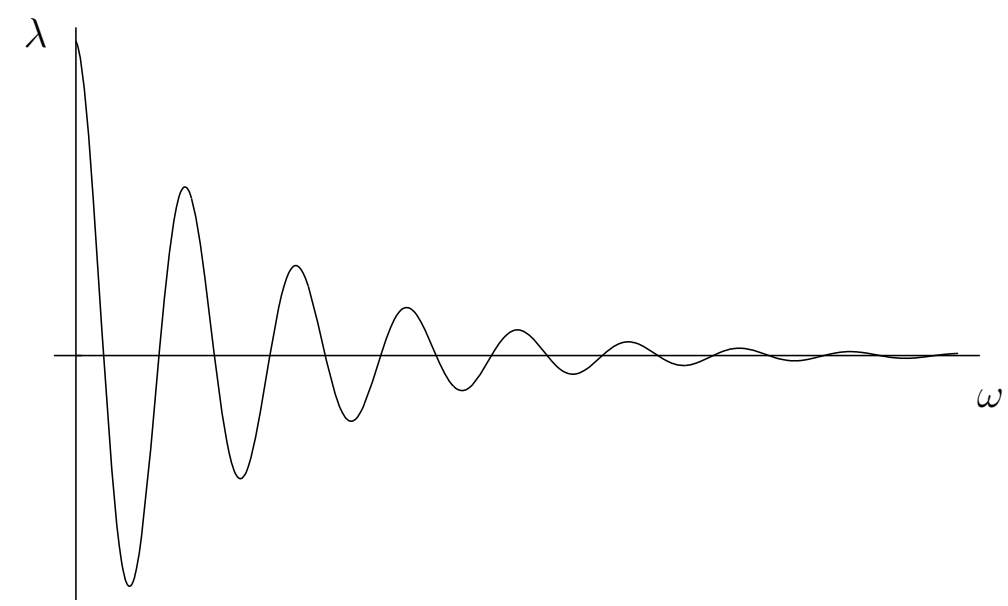

Figure 1. Snaking curve for symmetric $N$-homoclinic orbits.

This paper can be seen as a follow-up to [10], where we discussed one-homoclinic orbits near an EE cycle. There it has also be shown that $p_{2}$ has to be of saddle-focus type, in order to find snaking behaviour of one-homoclinic orbits. Furthermore, it has been shown there that $p_{1}$ has to be of saddle-focus type, too, if $N$-homoclinic orbits to this equilibrium are to exist.

Similar to the procedure in $[10]$ we will use Lin's method $[8,13]$ to derive bifurcation equations for $N$-homoclinic orbits near the cycle. The general setup will be introduced in Section 3. Under certain genericity assumptions it will be shown in Section 4 that the cycle is accompanied by a multitude of $N$ homoclinic orbits, which exist on snaking curves.

Before the general bifurcation analysis, however, we will illustrate the problem we are interested in by numerical results for a generalized Swift-Hohenberg equation in the next Section.

\section{$2 \quad N$-pulses in a generalized Swift-Hohenberg equation}

We consider the generalized Swift-Hohenberg equation studied in [6]

$$
\frac{\partial u}{\partial t}=r u-\left(\partial_{x}^{2}+q_{c}^{2}\right)^{2} u+v u^{2}-g u^{3} .
$$

Stationary localised solutions of this equation, that is, homoclinic solutions to 0 of the fourth order equation

$$
u^{\prime \prime \prime \prime}+2 q_{c}^{2} u^{\prime \prime}+\left(q_{c}^{4}-r\right) u-v u^{2}+g u^{3}=0
$$

have been discussed in detail in [1]. In particular, several snaking scenarios have been found to occur in (1). 


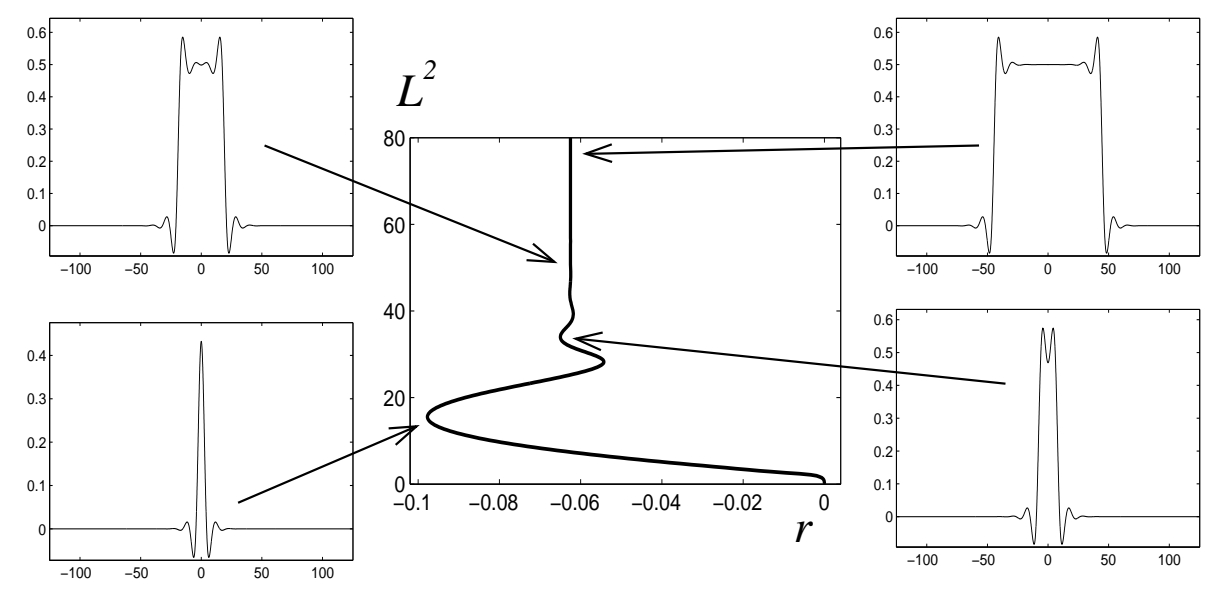

Figure 2. Snaking curve for one-homoclinic orbits of (1).

We stress the fact that Equation (1) is reversible. This means that there is a linear involution $R$ such that the corresponding first order system is invariant under the transformation $\left(\left(u, u^{\prime}, u^{\prime \prime}, u^{\prime \prime \prime}\right), x\right) \mapsto\left(R\left(u, u^{\prime}, u^{\prime \prime}, u^{\prime \prime \prime}\right),-x\right)$. Here the involution $R$ is defined by $\left(u, u^{\prime}, u^{\prime \prime}, u^{\prime \prime \prime}\right) \mapsto\left(u,-u^{\prime}, u^{\prime \prime},-u^{\prime \prime \prime}\right)$. The reversibility plays an important role for the dynamics of (1). In particular, it leads to the robust existence of those homoclinic solutions, for which $u$ is an even function. As is common, we will call such solutions symmetric. We refer to Section 3 for more general comments about reversible systems.

Note that in addition to being reversible, Equation (1) is also conservative and preserves a first integral. However, we will both in our computations for (1) and in the general analysis afterwards focus on symmetric homoclinics, and for these the reversibility of (1) is the most important property.

Following computations in [1], we set $q_{c}=0.5, v=0.75$ and $g=1$ and consider (1) as an equation depending on $r$ only. Then the 0 equilibrium is a saddle focus for all $r<0$, i.e. the linear part of the vector field has a quadruple of complex eigenvalues. Furthermore, there are two additional equilibria $u_{ \pm}=(3 \pm \sqrt{5+64 r}) / 8$, if $r>-5 / 64$.

We compute symmetric homoclinic orbits to 0 by shooting for orbits in the unstable manifold of this equilibrium, which intersect the symmetry section $\operatorname{Fix}(R)=\left\{\left(u, u^{\prime}, u^{\prime \prime}, u^{\prime \prime \prime}\right): u^{\prime}=u^{\prime \prime \prime}=0\right\}$. The behaviour and bifurcations of these orbits under variation of $r$ are studied using the software package AUTO/HomCont [19].

Figure 2 shows a bifurcation diagram for a particular one-homoclinic solution $H_{1}$. In this diagram we plot the $L^{2}$-norm of $H_{1}$ against the parameter $r$. We see that $H_{1}$ emerges at $r=0$ in a local bifurcation of the 0 -equilibrium and then undergoes a sequence of fold bifurcation along a snaking curve, which accumulates at $r^{*}=-1 / 16$. 
Inspection of the solutions, contained in the accompanying boxes, shows that along the snaking curve the middle part of the homoclinic orbit approaches the equilibrium $u_{+}$, and indeed a heteroclinic cycle $\Gamma$ between 0 and $u_{+}$is found to exist at $r^{*}$, see also [1]. Note that $\Gamma$ is invariant under $R$ - it is the limit of symmetric homoclinic orbits.

Remark. In all of the numerically obtained diagrams in this paper we plot the $L^{2}$-norm of the solution $u$ against the parameter, because this solution measure is numerically convenient. We note, however, that this norm is also directly related to the transition times, which will be used in the general analysis (compare with Figure 1). Indeed, the part of the solution that is close to $u_{+}$is the one that predominantly contributes to its $L^{2}$-norm.

Note that for $r=r^{*}$ there exist infinitely many homoclinic orbits to 0. Since 0 is of saddle-focus type for that parameter value, the results by Härterich [4] suggest the existence of $N$-homoclinic orbits to 0 . These $N$-homoclinic orbits are computed using a homoclinic branch-switching method developed in [12]. We find a multitude of symmetric $N$-homoclinic orbits and only consider a few of them with $N=2,3$ here.

Figure 3 contains a bifurcation diagram for two symmetric 2-homoclinic orbits existing near the cycle $\Gamma$. As before we plot the $L^{2}$-norm against the parameter $r$ and find a snaking curve for each orbit, which accumulates at $r=r^{*}$. Note that the snaking curves in the diagram stop at some finite norm. This is caused by numerical difficulties in continuing the solutions.

Some solutions along the green curve are shown to the right of the diagram. As for $H_{1}$ we find that along the snaking curve the pulses widen, and their middle parts approach $u_{+}$. It is interesting to observe that the central part of the solutions between the pulses remains unchanged.

We note that the diagram in Figure 3 shows only half of the $L^{2}$-norm of the 2homoclinic solutions, in order to allow for comparison with the snaking curve for the 1-homoclinic orbit. (The snaking curve for $H_{1}$ is shown in grey.) The green snaking curve is not close to the 1-homoclinic snaking curve, but we find a much better approximation by the red curve. This red curve corresponds to a 2-homoclinic solution for which the 2 pulses are more separated, compare also with the solution plots to the left of the diagram. This suggests that the snaking curves move closer together, if the pulses become more separated. However, we encounter numerical difficulties in finding and continuing such solutions.

Next we discuss numerical results for 3 -homoclinic obits near $\Gamma$. In Figure 4 we present two bifurcation diagrams for 3-homoclinic orbits near $H_{1}$. The orbits in this figure are created in saddle-node bifurcations and follow only parts of the snaking curve of $H_{1}$. Nevertheless, as the $L^{2}$-norm of the solutions increases, 


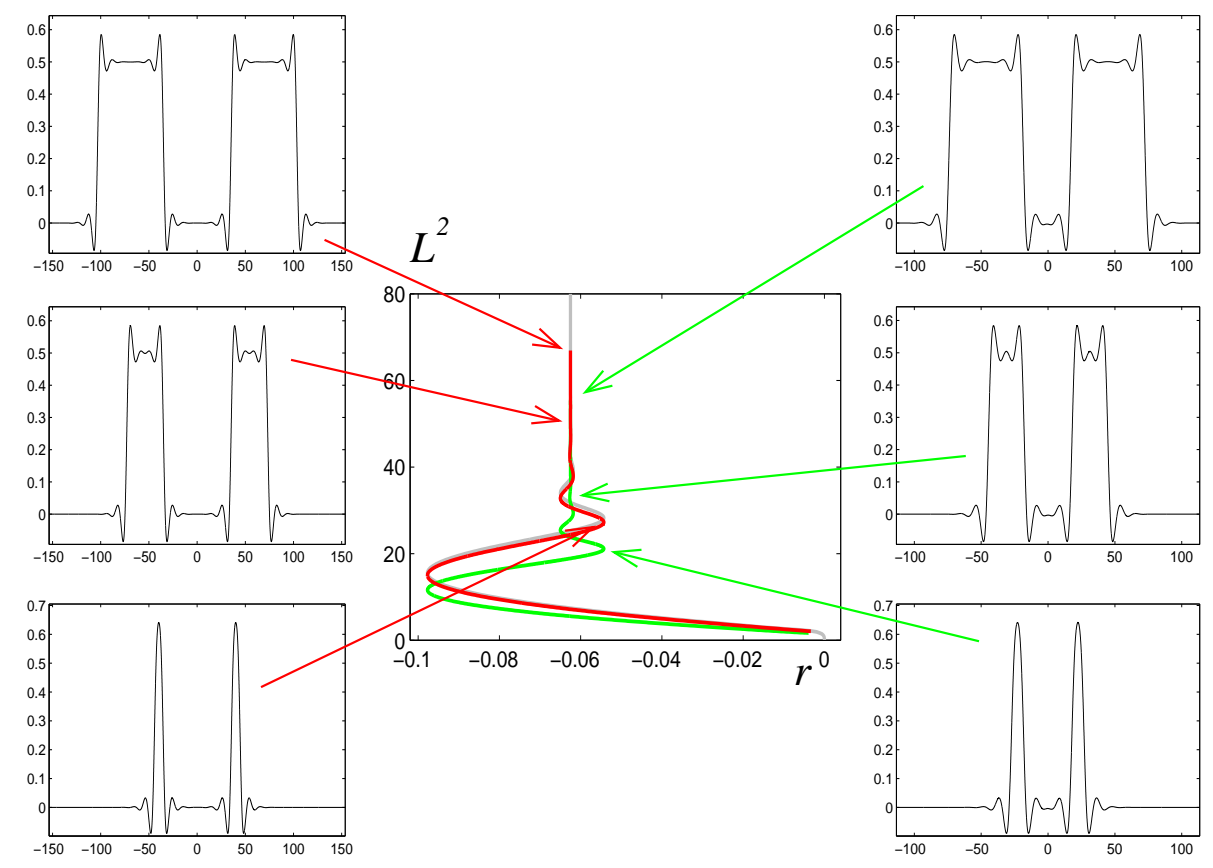

Figure 3. Snaking curves for 2-homoclinic orbits of (1).

the curves approach the parameter value $r^{*}$.

But now the solution plots show that there are two different types of behaviour along the snaking curves. Along the red snaking curves the outer pulses develop additional oscillations, whereas the middle pulse hardly changes. On the other hand, for orbits on the green curve it is the middle pulse that spreads out, and the outer pulses remain unchanged. In fact, solutions along the green curve approach a 2-heteroclinic cycle between 0 and $u_{+}$. The existence of such cycles will be investigated in Section 4.4. We note that for both types of snaking the times separating the pulses are again virtually constant along all snaking curves.

As before, the $L^{2}$ norm of the 3-homoclinic orbits is rescaled in Figure 4 to make the snaking comparable with the one for $H_{1}$, whose snaking curve (in parts) is again shown in grey. It is important that the rescaling is performed as to accommodate the behaviour along the red curves, that is, we plot an approximation to the $L^{2}$-norm of one of the outer pulses. This explains why there is a better match of the red and grey curves.

In summary, we have found 2- and 3-homoclinic orbits near $H_{1}$, which mirror the behaviour of this one-homoclinic solution in that they exist on snaking curves, along which certain pulses become wider and approach a steady state $u_{+}$. Furthermore, we expect to find different types of behaviour along snaking curves for $N$-homoclinic orbits with $N>2$. 

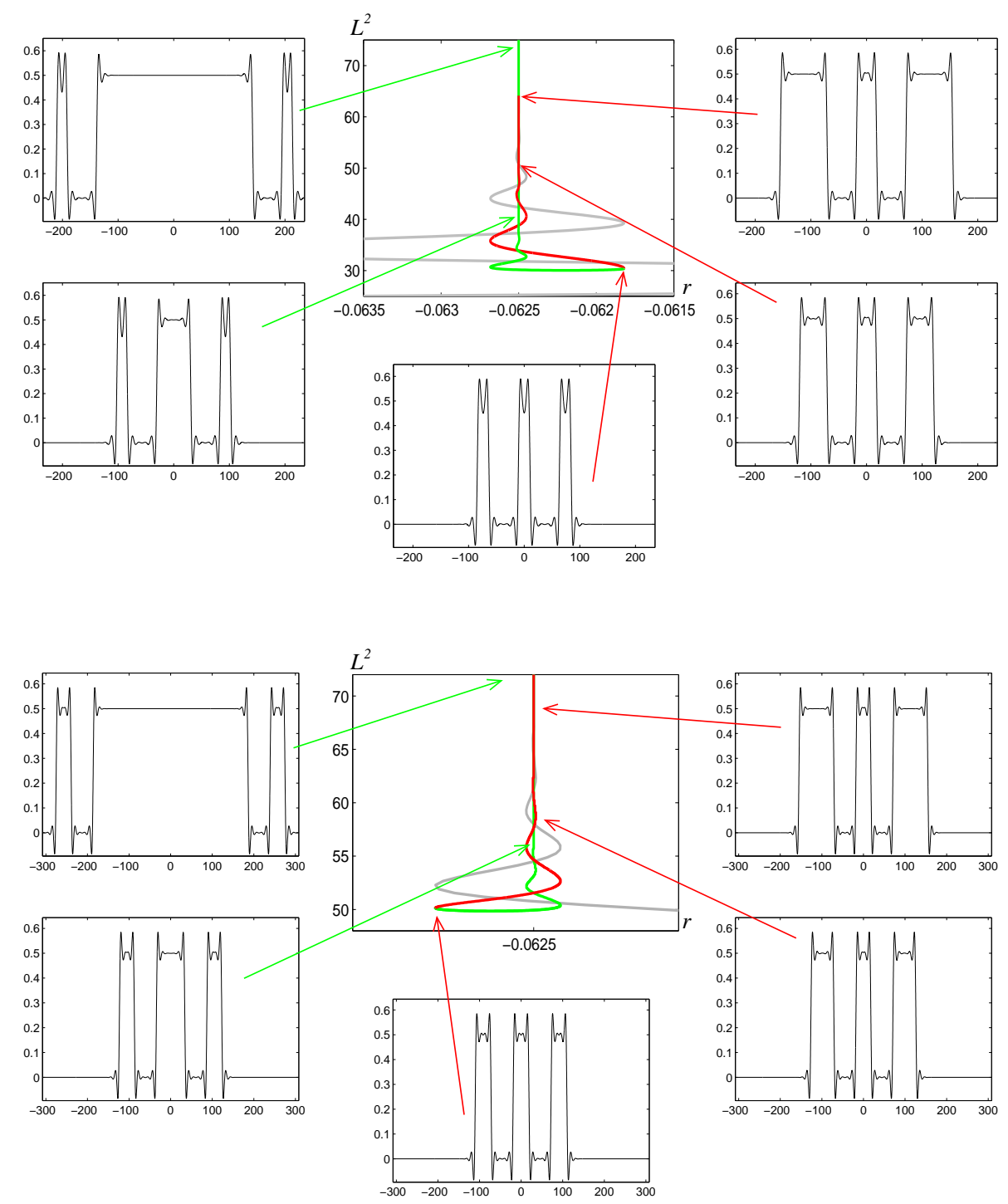

Figure 4. Two bifurcation diagrams for 3-homoclinic orbits near the heteroclinic cycle. Two different types of snaking behaviour are encountered along the red and green curves, respectively.

\section{$3 \quad$ Notations and setup}

We aim to understand the numerical results above in a more general context and are thus interested in bifurcations from a heteroclinic cycle between two symmetric equilibria of saddle-focus type in the class of time-reversible systems. Let us describe this configuration in detail.

We consider a system of ordinary differential equations

$$
\dot{x}=f(x, \lambda), \quad x \in \mathbb{R}^{2 n}, \lambda \in \mathbb{R}
$$

with a smooth vector field $f$, which is assumed to be (time-)reversible, that 
is, the vector fields anticommutes with some linear involution $R$ :

$$
R f(x, \lambda)=-f(R x, \lambda) .
$$

In a reversible system the image $R X$ of an orbit $X$ is also an orbit. Orbits, for which $R X=X$, are called symmetric. It is well known that orbits of a reversible system are symmetric if and only if they intersect the fixed space $\operatorname{Fix}(R):=\left\{x \in \mathbb{R}^{2 n}: R x=x\right\}$ of the involution $R$. We refer to $[11,16]$ for a collection of fundamental results about reversible systems.

We will be concerned with bifurcations from a heteroclinic cycle $\Gamma$ in (2). More precisely, $\Gamma$ is a collection of two equilibria $p_{1}, p_{2}$ and heteroclinic orbits connecting $p_{1}$ to $p_{2}$ and $p_{2}$ to $p_{1}$ in this order respectively. Let us first discuss the equilibria.

We assume that (2) possesses two symmetric equilibria of saddle-focus type. More precisely, we assume that there are $p_{1}, p_{2} \in \mathrm{Fix}(R)$, such that

$$
f\left(p_{k}, 0\right)=0, \quad k=1,2,
$$

and the stable spectrum of $D_{x} f\left(p_{k}, 0\right)$ has the structure

$$
\sigma^{s}\left(D_{x} f\left(p_{k}, 0\right)\right)=\left\{-\mu_{k}(0) \pm \phi_{k}(0) i\right\} \cup \sigma_{k}^{s s}, \quad \text { with } \mu_{k}(0), \phi_{k}(0)>0 .
$$

In Equation (3), $\sigma_{k}^{s s}$ denotes the strong stable spectrum, such that $\Re \mu<-\mu_{k}$ for all $\mu \in \sigma_{k}^{s s}$. Moreover, the principal eigenvalues $-\mu_{k}(0) \pm \phi_{k}(0) i$ are assumed to be simple. Due to the symmetry of the equilibria $p_{k}$ we find for the unstable spectrum that

$$
\sigma^{u}\left(D_{x} f\left(p_{k}, 0\right)\right)=-\sigma^{s}\left(D_{x} f\left(p_{k}, 0\right)\right) .
$$

By hyperbolicity the equilibria will persist as symmetric equilibria for small $\lambda$, and thus, with no loss of generality, we may assume that $f\left(p_{k}, \lambda\right)=0$ for all $\lambda$. Furthermore, the leading eigenvalues of the linearized vector field vary smoothly with $\lambda$; this means that both $\mu_{k}(\cdot)$ and $\phi_{k}(\cdot)$ are smooth functions of $\lambda$.

Let $W^{s(u)}\left(p_{k}, \lambda\right)$ denote the (un-)stable manifold of $p_{k}$ with respect to $f\left(p_{k}, \lambda\right)$. Again, due to the symmetry and hyperbolicity of the equilibria these manifolds are $n$-dimensional, see also (4). Moreover, reversibility implies $W^{s}\left(p_{k}, \lambda\right)=$ $R W^{u}\left(p_{k}, \lambda\right)$.

Finally, we assume the existence of a heteroclinic orbit $\Gamma_{1}=\left\{\gamma_{1}(t): t \in \mathbb{R}\right\}$ connecting $p_{1}$ to $p_{2}$ for $\lambda=0$. By reversibility, this orbit is part of a heteroclinic cycle $\Gamma$, together with $\Gamma_{2}=R \Gamma_{1}$ and the equilibria $p_{1}$ and $p_{2}$. Our analysis will require certain non-degeneracy conditions to be fulfilled. These will be imposed on $\Gamma_{1}$, and reversibility ensures that they are fulfilled along $\Gamma_{2}$, too. 
Firstly, we assume $\Gamma_{1}$ to be non-degenerate, that is we assume

$$
\operatorname{dim}\left(T_{\gamma_{1}(0)} W^{u}\left(p_{1}, 0\right) \cap T_{\gamma_{1}(0)} W^{s}\left(p_{2}, 0\right)\right)=1,
$$

where $T_{q} M$ denotes the tangent space of a manifold $M$ at the point $q$. As a consequence of (5), the equation $\dot{v}=-D_{x} f\left(\gamma_{1}(t), 0\right)^{*} v$ has a unique bounded solution $\psi_{1}$. We assume that both $\gamma_{1}$ and $\psi_{1}$ converge along the leading directions to the equilibria and zero, respectively, that is, we assume

$$
\begin{array}{lc}
\lim _{t \rightarrow-\infty} e^{\mu_{1} t}\left\|\gamma_{1}(t)-p_{1}\right\| \neq 0, & \lim _{t \rightarrow \infty} e^{\mu_{2} t}\left\|\gamma_{1}(t)-p_{2}\right\| \neq 0, \\
\lim _{t \rightarrow-\infty} e^{\mu_{2} t}\left\|\psi_{1}(t)\right\| \neq 0, & \lim _{t \rightarrow \infty} e^{\mu_{1} t}\left\|\psi_{1}(t)\right\| \neq 0 .
\end{array}
$$

Conditions (6) and (7) are known as non-orbit flip and non-inclination flip conditions, respectively [14]; see also [9] for an equivalent geometric statement.

Finally, we also assume a generic unfolding of the heteroclinic connection $\Gamma_{1}$, which should break up under variation of the parameter $\lambda$. This can be ensured by assuming that a Melnikov integral $M$ does not vanish, [14]:

$$
M:=\int_{\infty}^{\infty}\left\langle\psi_{1}(t), D_{\lambda} f\left(\gamma_{1}(t), 0\right)\right\rangle d t \neq 0
$$

\subsection{The main result}

We are interested in $N$-homoclinic orbits to $p_{1}$ that lie in a sufficiently small neighbourhood of the cycle $\Gamma$. Our main results reads as follows:

Theorem 3.1 Consider (2) near the heteroclinic cycle $\Gamma$ under the conditions set up in Section 3. Let $N \geq 1$ be fixed.

At $\lambda=0$ there exist countably many symmetric $N$-homoclinic orbits $\mathcal{H}$ to $p_{1}$ which can be continued on a snaking curve $\lambda_{\mathcal{H}}(\cdot)$ defined on $\left(\Omega_{\mathcal{H}}, \infty\right)$. The functions $\lambda_{\mathcal{H}}$ have countably infinitely many zeros, and $\left|\lambda_{\mathcal{H}}(\omega)\right|$ tends exponentially fast to zero as $\omega$ tends to infinity.

The graphs of $\lambda_{\mathcal{H}}$ are depicted in Figure 1. As already mentioned we will prove Theorem 3.1 for the case, where $\omega$ is the length of stay of the first passage of $\mathcal{H}$ near $p_{2}$. For a precise definition of $\omega$ we refer to Section 4. However, the numerical results for 3-homoclinic orbits of Equation 1 demonstrate that there are other possibilities for the choice of $\omega$. We will discuss these issues briefly in Section 4.4.

Note that for $N=1$ the theorem has already been proved in [10]. In this case all symmetric 1-homoclinic orbits (to $p_{1}$ ) lie on the same snaking curve. 
For $N>1$ it turns out that the length of all the other passages near $p_{1}$ and $p_{2}$ remain bounded as $\omega$ tends to infinity. This phenomenon can also be observed in the numerical bifurcation diagrams, see for example Figure 4. Along the red branch in that Figure, the outer pulses (representing the first and last passage near $p_{2}$ ) become and wider as the $L^{2}$ norm increases, while both the inner pulse and the time-intervals, by which the pulses are separated, remain nearly unchanged.

In addition, for fixed $N$ there are infinitely many different snaking curves. For example, two $N$-homoclinic orbits $\mathcal{H}$ and $\mathcal{H}^{\prime}$ (which might exist for the same $\lambda)$ are not on the same snaking curve if their numbers of rotations around $p_{1}$ and $p_{2}$ are "too different".

\section{The analysis}

We will analyse the existence of $N$-homoclinic orbits to $p_{1}$ using Lin's method, $[13,8]$. In a first step we determine $N$-homoclinic Lin orbits near the cycle $\Gamma$. An $N$-homoclinic Lin orbit to $p_{1}$ is a piecewise continuous orbit that starts in the unstable manifold of $p_{1}$, follows the cycle $\Gamma$ and finishes after $N$ loops in the stable manifold of $p_{1}$. Thereby, the discontinuities are only allowed to lie in certain places and have well defined jump directions. Figure 5 shows an impression of a 2-homoclinic Lin orbit near $\Gamma$.

\subsection{Set up of the bifurcation equation}

In the following we work exclusively in a neighbourhood $\mathcal{U}$ of $\Gamma$. To define $N$-homoclinic Lin orbits precisely, let $\Sigma_{1}$ be a hyperplane intersecting $\Gamma_{1}$ transversally at $\gamma_{1}(0)$, and let $\Sigma_{2}:=R \Sigma_{1}$. We are concerned with four different types of partial orbits. Firstly, let $X^{-}=\left\{x^{-}(t): t \in(-\infty, 0]\right\}$, such that $x^{-}(0) \in \Sigma_{1} \cap W^{u}\left(p_{1}, \lambda\right)$, and $x^{-}(t) \notin \Sigma_{k}$ for all $t<0$. Similarly, let $X^{+}=\left\{x^{+}(t): t \in[0, \infty)\right\}$, such that $x^{+}(0) \in \Sigma_{2} \cap W^{s}\left(p_{1}, \lambda\right)$ and $x^{+}(t) \notin \Sigma_{k}$ for all $t>0$. Finally, for positive numbers $\omega_{2}^{i}, \omega_{1}^{j}$, we consider orbits $X_{2}^{i}=$ $\left\{x_{2}^{i}(t): t \in\left[0,2 \omega_{2}^{i}\right]\right\}$ and $X_{1}^{j}=\left\{x_{1}^{j}(t): t \in\left[0,2 \omega_{1}^{j}\right]\right\}$, such that

$$
\begin{aligned}
& x_{2}^{i}(0) \in \Sigma_{1}, x_{2}^{i}\left(2 \omega_{2}^{i}\right) \in \Sigma_{2}, \text { and } x_{2}^{i}(t) \notin \Sigma_{2} \text { for } t \in\left(0,2 \omega_{2}^{i}\right), \\
& x_{1}^{j}(0) \in \Sigma_{2}, x_{1}^{j}\left(2 \omega_{1}^{j}\right) \in \Sigma_{1}, \text { and } x_{1}^{j}(t) \notin \Sigma_{1} \text { for } t \in\left(0,2 \omega_{1}^{j}\right) .
\end{aligned}
$$

Now, we introduce a space $Z_{1} \subset T_{\gamma_{1}(0)} \Sigma_{1}$, complementary to $T_{\gamma_{1}(0)} W^{u}\left(p_{1}, 0\right)+$ $T_{\gamma_{1}(0)} W^{s}\left(p_{2}, 0\right)$. Note that because of $(5)$ we have $\operatorname{dim} Z_{1}=1$. Furthermore, 


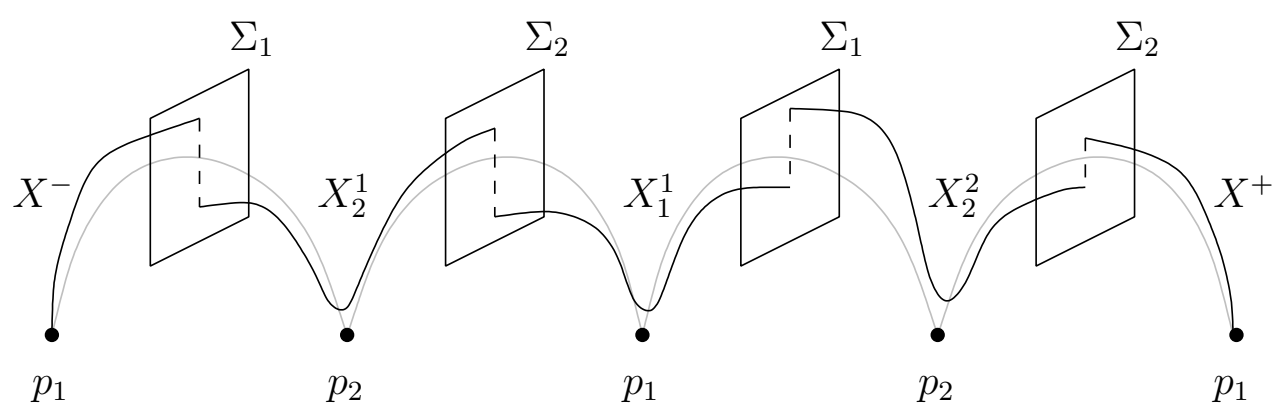

Figure 5. A 2-homoclinic Lin orbit near the cycle $\Gamma$. The original heteroclinic orbits are shown in grey. Note that for the purpose of illustration $\Gamma$ is shown as a heteroclinic chain.

let $Z_{2}=R Z_{1} \subset \Sigma_{2}$. Then a collection of partial orbits

$$
\mathcal{L}=\left(X^{-}, X_{2}^{1}, X_{1}^{1}, X_{2}^{2}, \ldots, X_{1}^{N-1}, X_{2}^{N}, X^{+}\right)
$$

is called an $N$-homoclinic Lin orbit to $p_{1}$ if the jump between two consecutive partial orbits is parallel to $Z_{1}$ or $Z_{2}$, respectively.

Note that the lower index $k$ of $X_{k}^{i}$ indicates that this partial orbit passes $p_{k}$ while the upper index $i$ counts the number of passages past the equilibrium $p_{k}$. The indices in the corresponding quantities $x_{k}^{i}$ and $\omega_{k}^{i}$ have the same meaning.

Lin orbits can be characterised by the times $\omega_{2}^{i}$ and $\omega_{1}^{j}$, and by the parameter $\lambda$. More precisely, we have the following result.

Lemma $4.1([13,8])$ There are positive numbers $\hat{\lambda}$ and $\hat{\omega}$, such that for each $|\lambda|<\hat{\lambda}$ and each sets $\boldsymbol{\omega}_{1}=\left\{\omega_{1}^{1}, \ldots, \omega_{1}^{N-1}\right\}$ and $\boldsymbol{\omega}_{2}=\left\{\omega_{2}^{1}, \ldots, \omega_{2}^{N}\right\}$ with

$$
\min \left\{\omega_{1}^{j}, \omega_{2}^{i}: j=1, \ldots, N-1, i=1, \ldots, N\right\}>\hat{\omega}
$$

there exists a unique $N$-homoclinic Lin orbit $\mathcal{L}\left(\boldsymbol{\omega}_{1}, \boldsymbol{\omega}_{2}, \lambda\right)$ as introduced above.

The detection of $N$-homoclinic orbits near $\Gamma$ now amounts to finding those Lin orbits without discontinuities (jumps), which are given by

$$
\begin{aligned}
& \Xi_{1}^{1}=x^{-}(0)-x_{2}^{1}(0) \\
& \Xi_{1}^{i}=x_{1}^{i-1}\left(2 \omega_{1}^{i-1}\right)-x_{2}^{i}(0), i=2, \ldots, N, \\
& \Xi_{2}^{i}=x_{2}^{i}\left(2 \omega_{2}^{i}\right)-x_{1}^{i}(0), i=1, \ldots, N-1, \\
& \Xi_{2}^{N}=x_{2}^{N}\left(2 \omega_{2}^{N}\right)-x^{+}(0) .
\end{aligned}
$$

The lower index $k$ of $\Xi_{k}^{i}$ indicates in which cross-section the jump takes place, and the upper index $i$ counts the jumps in these cross-sections. 
Setting $\boldsymbol{\Xi}_{1}=\left(\Xi_{1}^{1}, \ldots, \Xi_{1}^{N}\right)$ and $\boldsymbol{\Xi}_{2}=\left(\Xi_{2}^{1}, \ldots, \Xi_{2}^{N}\right)$ we find by Lemma 4.1 that $\boldsymbol{\Xi}_{i}=\boldsymbol{\Xi}_{i}\left(\boldsymbol{\omega}_{1}, \boldsymbol{\omega}_{2}, \lambda\right)$. In order to detect actual $N$-homoclinic orbits to $p_{1}$ we have to solve the bifurcation equations

$$
\boldsymbol{\Xi}_{1}\left(\boldsymbol{\omega}_{1}, \boldsymbol{\omega}_{2}, \lambda\right)=0, \quad \boldsymbol{\Xi}_{2}\left(\boldsymbol{\omega}_{1}, \boldsymbol{\omega}_{2}, \lambda\right)=0
$$

Within the general framework of Lin's method we can derive expressions for the terms $\boldsymbol{\Xi}_{k}, k=1,2$. We will do this for $\boldsymbol{\Xi}_{1}$ only, since it turns out that only this is needed when we focus on symmetric orbits.

For the jumps $\Xi_{1}^{i}=\Xi_{1}^{i}\left(\boldsymbol{\omega}_{1}, \boldsymbol{\omega}_{2}, \lambda\right)$ it has been shown in $[13,8]$ that

$$
\Xi_{1}^{i}\left(\boldsymbol{\omega}_{1}, \boldsymbol{\omega}_{2}, \lambda\right)=\xi^{\infty}(\lambda)+\xi_{1}^{i}\left(\boldsymbol{\omega}_{1}, \boldsymbol{\omega}_{2}, \lambda\right),
$$

where $\xi^{\infty}(\lambda)$ measures the splitting of $W^{u}\left(p_{1}, \lambda\right)$ and $W^{s}\left(p_{2}, \lambda\right)$ in the direction $Z_{1}$. Obviously, $\xi^{\infty}(0)=0$, and according to assumption (8) we have $D \xi^{\infty}(0) \neq$ 0 . Hence, with no loss of generality we may assume that

$$
\xi^{\infty}(\lambda)=\lambda
$$

While $\xi^{\infty}$ only depends on $\lambda$, the terms $\xi_{1}^{i}$ measure the influence of the finite transition times between the $\Sigma_{i}$, too. The leading terms in the $\xi_{1}^{i}$ depend on the asymptotic behaviour near the equilibria $p_{1}$ and $p_{2}$. Applying general results from $[13,8]$ we find

Lemma 4.2 Assuming the non-degeneracy conditions (3), (6) and (7) the jumps $\xi_{1}^{i}$ have the following representation:

$$
\begin{aligned}
& \xi_{1}^{1}\left(\boldsymbol{\omega}_{1}, \boldsymbol{\omega}_{2}, \lambda\right)=L_{2}\left(\omega_{2}^{1}, \lambda\right)+\mathcal{R}^{1}, \\
& \xi_{1}^{i}\left(\boldsymbol{\omega}_{1}, \boldsymbol{\omega}_{2}, \lambda\right)=L_{1}\left(\omega_{1}^{i-1}, \lambda\right)+L_{2}\left(\omega_{2}^{i}, \lambda\right)+\mathcal{R}^{i}, \quad i=2, \ldots, N,
\end{aligned}
$$

where

$$
\begin{aligned}
& L_{k}(\omega, \lambda):=c_{k}(\lambda) e^{-2 \mu_{k}(\lambda) \omega} \sin \left(2 \phi_{k}(\lambda) \omega+\varphi_{k}(\lambda)\right), \quad k=1,2, \\
& \mathcal{R}^{1}=\mathcal{O}\left(e^{-2 \alpha \mu_{2}(\lambda) \omega_{2}^{1}}\right), \quad \mathcal{R}^{i}=\mathcal{O}\left(e^{-2 \alpha \mu_{1}(\lambda) \omega_{1}^{i-1}}\right)+\mathcal{O}\left(e^{-2 \alpha \mu_{2}(\lambda) \omega_{2}^{i}}\right),
\end{aligned}
$$

$i=2, \ldots, N$. Here $\alpha$ is some real number greater than one. The quantities $c_{1}$, $c_{2}$ and $\varphi_{1}, \varphi_{2}$ depend smoothly on $\lambda$, and we have $c_{1}(0) \neq 0, c_{2}(0) \neq 0$.

Also from $[13,8]$ we know that the jumps $\xi_{k}^{i}$ are differentiable, and moreover we have the following estimates of the derivatives:

Lemma 4.3 Under the assumptions of Lemma 4.2 the mappings $\xi_{1}^{i}: \mathbb{R}^{N-1} \times$ $\mathbb{R}^{N} \times \mathbb{R} \rightarrow \mathbb{R}$ are smooth and the partial derivatives $D_{j} \xi_{1}^{i}, j \in\{1,2,3\}$ can be 
estimated as follows:

$$
\begin{aligned}
D_{j} \xi_{1}^{1}\left(\boldsymbol{\omega}_{1}, \boldsymbol{\omega}_{2}, \lambda\right)= & D_{j} L_{2}\left(\omega_{2}^{1}, \lambda\right)+o\left(e^{-2 \mu_{2}(\lambda) \omega_{2}^{1}}\right), \\
D_{j} \xi_{1}^{i}\left(\boldsymbol{\omega}_{1}, \boldsymbol{\omega}_{2}, \lambda\right)= & D_{j}\left(L_{1}\left(\omega_{1}^{i-1}, \lambda\right)+L_{2}\left(\omega_{2}^{i}, \lambda\right)\right) \\
& +\mathcal{O}\left(e^{-2 \alpha \mu_{1}(\lambda) \omega_{1}^{i-1}}\right)+\mathcal{O}\left(e^{-2 \alpha \mu_{2}(\lambda) \omega_{2}^{i}}\right),
\end{aligned}
$$

$i=2, \ldots, N$. Again $\alpha$ is some real number greater than one.

In the following we will focus on symmetric $N$-homoclinic orbits to $p_{1}$. Those orbits correspond to symmetric $N$-homoclinic Lin orbits to $p_{1}$, which are characterised by

$$
\begin{aligned}
& X^{-}=R X^{+}, \quad X_{1}^{i}=R X_{1}^{N-i}, i=1, \ldots,\lfloor N / 2\rfloor \quad \text { and } \\
& X_{2}^{i}=R X_{2}^{N+1-i}, i=1, \ldots,\lfloor(N+1) / 2\rfloor,
\end{aligned}
$$

where $\lfloor r\rfloor$ denotes the integer part of the real number $r$. If $N$ is even, then the partial orbit $X_{1}^{N / 2}$ is symmetric and the $N$-homoclinic Lin orbit intersects Fix $R$ near $p_{1}$, while for $N$ odd $X_{2}^{(N+1) / 2}$ is symmetric and the $N$-homoclinic Lin orbit intersects Fix $R$ near $p_{2}$.

In particular, this implies that

$$
\omega_{1}^{i}=\omega_{1}^{N-i}, i=1, \ldots\left\lfloor\frac{N}{2}\right\rfloor \quad \text { and } \quad \omega_{2}^{i}=\omega_{2}^{N+1-i}, i=1, \ldots,\left\lfloor\frac{N+1}{2}\right\rfloor .
$$

Taking these particularities of the transition times into consideration we will henceforth write

$$
\omega:=\omega_{2}^{1}, \quad \boldsymbol{\omega}:=\left(\omega_{1}^{1}, \ldots, \omega_{1}^{\left\lfloor\frac{N}{2}\right\rfloor}, \omega_{2}^{2}, \ldots, \omega_{2}^{\left\lfloor\frac{N+1}{2}\right\rfloor}\right) .
$$

Furthermore the symmetry of an $N$-homoclinic Lin orbit implies

$$
\Xi_{1}^{i}=R \Xi_{2}^{N+1-i}, i=1, \ldots, N
$$

Hence, $\boldsymbol{\Xi}_{1}=0$ if and only if $\boldsymbol{\Xi}_{2}=0$, and the bifurcation equation for symmetric $N$-homoclinic orbits to $p_{1}$ reads

$$
\boldsymbol{\Xi}(\omega, \boldsymbol{\omega}, \lambda):=\boldsymbol{\Xi}_{1}\left(\boldsymbol{\omega}_{1}, \boldsymbol{\omega}_{2}, \lambda\right)=0
$$

In what follows we just write $\Xi^{i}$ instead of $\Xi_{1}^{i}$. With these notations we have:

$$
\begin{aligned}
& \Xi^{1}=\lambda+L_{2}\left(\omega_{2}^{1}, \lambda\right)+\mathcal{R}^{1} \\
& \Xi^{i}=\lambda+L_{1}\left(\omega_{1}^{i-1}, \lambda\right)+L_{2}\left(\omega_{2}^{i}, \lambda\right)+\mathcal{R}^{i}, \quad i=2, \ldots,\left\lfloor\frac{N+1}{2}\right\rfloor, \\
& \Xi^{i}=\lambda+L_{1}\left(\omega_{1}^{N+1-i}, \lambda\right)+L_{2}\left(\omega_{2}^{N+1-i}, \lambda\right)+\mathcal{R}^{i}, \quad i=\left\lfloor\frac{N+1}{2}\right\rfloor+1, \ldots, N .
\end{aligned}
$$


Note that all $\Xi^{i}$ and $\mathcal{R}^{i}$ depend on $(\omega, \boldsymbol{\omega}, \lambda)$.

\subsection{Reformulation of the bifurcation equation}

We define

$$
r_{k}^{i}:=e^{-2 \mu_{k}(0) \omega_{k}^{i}}, \quad r:=r_{2}^{1}, \quad \mathbf{r}:=\left(r_{1}^{1}, \ldots, r_{1}^{\left\lfloor\frac{N}{2}\right\rfloor}, r_{2}^{2}, \ldots, r_{2}^{\left\lfloor\frac{N+1}{2}\right\rfloor}\right) .
$$

We want to emphasize that by definition all $r$ and as well as all components of $\mathbf{r}$ are greater than zero.

With that we write the jumps as quantities depending on $(r, \mathbf{r}, \lambda)$ :

$$
\hat{\boldsymbol{\Xi}}(r, \mathbf{r}, \lambda)=\left(\hat{\Xi}^{1}(r, \mathbf{r}, \lambda), \ldots, \hat{\Xi}^{N}(r, \mathbf{r}, \lambda)\right):=\boldsymbol{\Xi}(\omega(r), \boldsymbol{\omega}(\mathbf{r}), \lambda) .
$$

Corollary 4.4 The $(r, \mathbf{r})$-dependent jumps reads as follows:

$$
\begin{aligned}
& \hat{\Xi}^{1}=\lambda+\hat{\mathcal{R}}^{1} \\
& \hat{\Xi}^{i}=\lambda+\hat{L}_{1}\left(r_{1}^{i-1}, \lambda\right)+\hat{L}_{2}\left(r_{2}^{i}, \lambda\right)+\hat{\mathcal{R}}^{i}, \quad i=2, \ldots,\left\lfloor\frac{N+1}{2}\right\rfloor, \\
& \hat{\Xi}^{i}=\lambda+\hat{L}_{1}\left(r_{1}^{N+1-i}, \lambda\right)+\hat{L}_{2}\left(r_{2}^{N+1-i}, \lambda\right)+\hat{\mathcal{R}}^{i}, \quad i=\left\lfloor\frac{N+1}{2}\right\rfloor+1, \ldots, N-1, \\
& \hat{\Xi}^{N}=\lambda+\hat{L}_{1}\left(r_{1}^{1}, \lambda\right)+\hat{\mathcal{R}}^{N} .
\end{aligned}
$$

where $\hat{\mathcal{R}}^{i}=\hat{\mathcal{R}}^{i}(r, \mathbf{r}, \lambda)$, and

$$
\hat{L}_{k}(s, \lambda):=c_{k}(\lambda) s^{\frac{\mu_{k}(\lambda)}{\mu_{k}(0)}} \sin \left(-\frac{\phi_{k}(\lambda)}{\mu_{k}(0)} \ln s+\varphi_{k}(\lambda)\right), \quad k=1,2,
$$

and, with some $\alpha>1$,

$$
\begin{aligned}
& \hat{\mathcal{R}}^{1}=\hat{L}_{2}(r, \lambda)+\mathcal{O}\left(r^{\alpha}\right) \\
& \hat{\mathcal{R}}^{i}=\mathcal{O}\left(\left(r_{1}^{i-1}\right)^{\alpha}\right)+\mathcal{O}\left(\left(r_{2}^{i}\right)^{\alpha}\right), \quad i=2, \ldots, N-1 \\
& \hat{\mathcal{R}}^{N}=\hat{L}_{2}(r, \lambda)+\mathcal{O}\left(\left(r_{1}^{N-1}\right)^{\alpha}\right)+\mathcal{O}\left(\left(r_{2}^{N}\right)^{\alpha}\right) .
\end{aligned}
$$

And similarly we get estimates for the derivatives of the residual terms $\hat{\mathcal{R}}^{i}=$ $\hat{\mathcal{R}}^{i}(r, \mathbf{r}, \lambda)$ from Lemma 4.3 :

Corollary 4.5 The statement of Lemma 4.3 for the $(r, \mathbf{r})$-dependent jumps 
reads as follows:

$$
\begin{aligned}
& D_{1} \hat{\mathcal{R}}^{1}=D_{1} \hat{L}_{2}(r, \lambda)+\mathcal{O}\left(r^{\alpha-1}\right), \quad D_{2} \hat{\mathcal{R}}^{1}=\mathcal{O}\left(r^{\alpha}\right), \\
& D_{3} \hat{\mathcal{R}}^{1}=D_{3} \hat{L}_{2}(r, \lambda)+\mathcal{O}\left(r^{\alpha}\right), \\
& D_{2} \hat{\mathcal{R}}^{i}=\mathcal{O}\left(\left(r_{1}^{i-1}\right)^{\alpha-1}\right)+\mathcal{O}\left(\left(r_{2}^{i}\right)^{\alpha-1}\right), \quad i=2, \ldots, N, \\
& D_{3} \hat{\mathcal{R}}^{i}=\mathcal{O}\left(\left(r_{1}^{i-1}\right)^{\alpha}\right)+\mathcal{O}\left(\left(r_{2}^{i}\right)^{\alpha}\right), \quad i=2, \ldots, N-1, \\
& D_{3} \hat{\mathcal{R}}^{N}=D_{3} \hat{L}_{2}(r, \lambda)+\mathcal{O}\left(\left(r_{1}^{N-1}\right)^{\alpha}\right)+\mathcal{O}\left(\left(r_{2}^{N}\right)^{\alpha}\right) .
\end{aligned}
$$

Our goal is to rewrite the bifurcation equation $\hat{\boldsymbol{\Xi}}=0$ as a fixed point equation. For this we introduce

$$
\begin{aligned}
& \hat{\mathbf{L}}(\mathbf{r}, \lambda):=\left(\hat{L}_{1}\left(r_{1}^{1}, \lambda\right), \ldots, \hat{L}_{1}\left(r_{1}^{\left\lfloor\frac{N}{2}\right\rfloor}, \lambda\right), \hat{L}_{2}\left(r_{2}^{2}, \lambda\right), \ldots, \hat{L}_{2}\left(r_{2}^{\left\lfloor\frac{N+1}{2}\right\rfloor}, \lambda\right)\right)^{T}, \\
& \hat{\mathcal{R}}:=\left(\hat{\mathcal{R}}^{1}, \ldots, \hat{\mathcal{R}}^{N}\right)^{T} .
\end{aligned}
$$

There is an invertible constant $(N \times N)$-matrix $\mathcal{M}$ such that

$$
\hat{\boldsymbol{\Xi}}(r, \mathbf{r}, \lambda)=\mathcal{M}\left(\begin{array}{c}
\hat{\mathbf{L}}(\mathbf{r}, \lambda) \\
\lambda
\end{array}\right)+\hat{\mathcal{R}}(r, \mathbf{r}, \lambda)
$$

The entries of $\mathcal{M}$ are either one or zero. To show that $\mathcal{M}$ is indeed nonsingular we simply compute its determinant by using Laplace's formula. The main observation in this respect is that $\mathcal{M}$ and each minor arising in this procedure has (exactly) one row whose entries but one are zeros (we always expand the corresponding determinant along that row). This finally yields that the determinant of $\mathcal{M}$ is some power of $(-1)$.

With that $\hat{\mathbf{\Xi}}(r, \mathbf{r}, \lambda)=0$ is equivalent to

$$
\left(\begin{array}{c}
\hat{\mathbf{L}}(\mathbf{r}, \lambda) \\
\lambda
\end{array}\right)=-\mathcal{M}^{-1} \hat{\mathcal{R}}(r, \mathbf{r}, \lambda)
$$

In the next step we rewrite (13) into a fixed point equation. For that we choose $\hat{\mathbf{r}}$ such that $\hat{\mathbf{L}}(\hat{\mathbf{r}}, 0)=0$. Note that there are infinitely many candidates for such $\hat{\mathbf{r}}$ which accumulate at zero. However, for either such $\hat{\mathbf{r}}$ the partial derivative w.r.t. the first variable $D_{1} \hat{\mathbf{L}}(\hat{\mathbf{r}}, 0)$ is an invertible diagonal matrix $\mathcal{D}$, and the absolute values of the entries in the diagonal are either $\left|\frac{c_{1}(0) \phi_{1}(0)}{\mu_{1}(0)}\right|$ or $\left|\frac{c_{2}(0) \phi_{2}(0)}{\mu_{2}(0)}\right|$. We want to emphasize that these quantities do not depend on the particular choice of $\hat{\mathbf{r}}$ (as long as $\hat{\mathbf{L}}(\hat{\mathbf{r}}, 0)=0$ ).

With that the Taylor expansion of $\hat{\mathbf{L}}$ at $(\mathbf{r}, \lambda)=(\hat{\mathbf{r}}, 0)$ with second order 
residual term $\hat{\mathbf{L}}_{r e s, \hat{\mathbf{r}}}$ reads

$$
\hat{\mathbf{L}}(\mathbf{r}, \lambda)=\mathcal{D}(\mathbf{r}-\hat{\mathbf{r}})+D_{2} \hat{\mathbf{L}}(\hat{\mathbf{r}}, 0) \lambda+\hat{\mathbf{L}}_{r e s, \hat{\mathbf{r}}}(\mathbf{r}, \lambda) .
$$

Combining (13) and (14) we find the following fixed point equation for $\mathbf{r}$ which is equivalent to $\hat{\boldsymbol{\Xi}}(r, \mathbf{r}, \lambda)=0$ :

$$
\begin{aligned}
\left(\begin{array}{l}
\mathbf{r} \\
\lambda
\end{array}\right) & =\left(\begin{array}{c}
\hat{\mathbf{r}}-\mathcal{D}^{-1}\left(D_{2} \hat{\mathbf{L}}(\hat{\mathbf{r}}, 0) \lambda+\hat{\mathbf{L}}_{r e s, \hat{\mathbf{r}}}(\mathbf{r}, \lambda)\right) \\
0
\end{array}\right)-(\mathcal{M} \hat{\mathcal{D}})^{-1} \hat{\mathcal{R}}(r, \mathbf{r}, \lambda) \\
& =: \mathcal{T}_{\hat{\mathbf{r}}}(r, \mathbf{r}, \lambda)
\end{aligned}
$$

where $\hat{\mathcal{D}}=\left(\begin{array}{ll}\mathcal{D} & 0 \\ 0 & 1\end{array}\right)$. The right-hand side $\mathcal{T}_{\hat{\mathbf{r}}}$ of this equation can be read as a mapping

$$
\mathcal{T}_{\hat{\mathbf{r}}}: \mathbb{R}_{+} \times \mathbb{R}_{+}^{N-1} \times \mathbb{R} \rightarrow \mathbb{R}_{+}^{N-1} \times \mathbb{R}
$$

The lower index "+" denotes the restriction to positive numbers.

\subsection{Proof of Theorem 3.1}

Our goal is to solve the reformulated bifurcation equation (15) for $\left(\mathbf{r}_{\hat{\mathbf{r}}}, \lambda_{\hat{\mathbf{r}}}\right)(r)$ near $(\mathbf{r}, \lambda)=(\hat{\mathbf{r}}, 0), r \in(0, \epsilon)$. We will do this by applying the Banach Fixed Point Theorem. Our strategy is as follows. First we construct for the "principal part"

$$
\left(\begin{array}{l}
\mathbf{r} \\
\lambda
\end{array}\right)=\left(\begin{array}{c}
\hat{\mathbf{r}}-\mathcal{D}^{-1}\left(D_{2} \hat{\mathbf{L}}(\hat{\mathbf{r}}, 0) \lambda+\hat{\mathbf{L}}_{r e s, \hat{\mathbf{r}}}(\mathbf{r}, \lambda)\right) \\
0
\end{array}\right)=: \mathcal{T}_{r e d, \hat{\mathbf{r}}}(r, \mathbf{r}, \lambda),
$$

of that equation a domain $\mathcal{C}_{\hat{\mathbf{r}}} \times \mathcal{B}_{\hat{\mathbf{r}}}$, which will be mapped contractively into itself by $\mathcal{T}_{\text {red } \hat{\mathbf{r}}}$. Then we make clear that for small $\hat{\mathbf{r}}$ this can be carried forward to $\mathcal{T}_{\hat{\mathbf{r}}}$.

In a first step we consider $\hat{L}_{k}-$ which represent the components of $\hat{\mathbf{L}}$. To simplify matter we omit the lower index $k$ in the further considerations:

$$
\hat{L}(s, \lambda):=c(\lambda) s^{\frac{\mu(\lambda)}{\mu(0)}} \sin \left(-\frac{\phi(\lambda)}{\mu(0)} \ln s+\varphi(\lambda)\right) .
$$

The zeros $s_{n}$ of $\hat{L}(\cdot, 0)$ are explicitely given by $s_{n}=e^{-\frac{\mu(0)}{\phi(0)}(n \pi-\varphi(0))}, \quad n \in \mathbb{Z}$. For our purpose we are only interested in those zeros which are close to $s=0$, so we may assume $n \in \mathbb{N}$, sufficiently large.

Let $\delta:=D_{1} \hat{L}\left(s_{n}, 0\right)$. Note that $\delta$ represents an element in the diagonal of $\mathcal{D}$, and that $|\delta|$ does not depend on $n$. By $\hat{L}_{r e s, n}$ we denote the second order 
residual term of the Taylor expansion of $\hat{L}$ at $\left(s_{n}, 0\right)$. We consider the following reduced fixed point equation:

$$
s=s_{n}-\delta^{-1}\left(D_{2} \hat{L}\left(s_{n}, 0\right) \lambda+\hat{L}_{r e s, n}(s, \lambda)\right)=: T_{n}(s, \lambda) .
$$

Let $B[s, \rho]$ be the closed ball centered at $s$ with radius $\rho$.

Lemma 4.6 There is a $\bar{\beta}>0$ such that for all $n \in \mathbb{N}$ there exists a positive real number $l_{s_{n}}$ such that for all $s \in B\left[s_{n}, \rho_{n}\right], \rho_{n}:=s_{n} \bar{\beta}$, and all $\lambda \in B\left[0, l_{s_{n}}\right]$

$$
\left|D_{1} T_{n}(s, \lambda)\right|<\frac{1}{3}, \quad\left|T_{n}(s, \lambda)-s_{n}\right|<\frac{2}{3} \rho_{n} .
$$

Proof. From (16) we read easily that $D_{1} T_{n}(s, \lambda)=\delta^{-1} D_{1} \hat{L}_{r e s, n}(s, \lambda)$. With $A(s, \lambda):=-\frac{\phi(\lambda)}{\mu(0)} \ln s+\varphi(\lambda)$ we get

$$
\hat{L}_{r e s, n}(s, \lambda)=c(\lambda) s^{\frac{\mu(\lambda)}{\mu(0)}} \sin (A(s, \lambda))-\delta\left(s-s_{n}\right)-D_{2} \hat{L}\left(s_{n}, 0\right) \lambda
$$

and therefore

$$
D_{1} \hat{L}_{r e s, n}(s, \lambda)=c(\lambda) s^{\frac{\mu(\lambda)}{\mu(0)}-1}\left[\frac{\mu(\lambda)}{\mu(0)} \sin (A(s, \lambda))-\frac{\phi(\lambda)}{\mu(0)} \cos (A(s, \lambda))\right]-\delta .
$$

Obviously $D_{1} \hat{L}_{r e s, n}\left(s_{n}, 0\right)=0$. Writing $s=s_{n} \beta$ one finds that there is a $\bar{\beta}$ and a $\tilde{l}_{s_{n}}$ such that for all $\beta \in[1-\bar{\beta}, 1+\bar{\beta}]$ and all $\lambda \in B\left[0, \tilde{l}_{s_{n}}\right]$

$$
\left|D_{1} \hat{L}_{r e s, n}\left(s_{n} \beta, \lambda\right)\right|<\frac{\delta}{3} .
$$

Note that for those $\beta$ the corresponding $s=s_{n} \beta$ belong to $B\left[s_{n}, \rho_{n}\right]$. This proves the assertion concerning $D_{1} T_{n}$.

It remains to show that $T_{n}$ maps $B\left[s_{n}, \rho_{n}\right]$ into itself. For that we first notice that

$$
\lim _{n \rightarrow \infty} D_{2} \hat{L}\left(s_{n}, 0\right)=0
$$

where we exploit that $\lim _{s \rightarrow+0} s \ln s=0$. So, in particular $D_{2} \hat{L}\left(s_{n}, 0\right)$ remains bounded. Similarly we find that $D_{2} \hat{L}_{r e s, n}(s, \lambda)$ remains bounded if $s$ and $\lambda$ are close to zero. The boundedness of $D_{2} \hat{L}_{\text {res,n }}$ together with the mean value theorem imply that there is a constant $C$ such that

$$
\begin{aligned}
\left|\hat{L}_{r e s, n}(s, \lambda)\right| & \leq \sup _{\substack{s \in B\left[s_{n}, \rho_{n}\right] \\
\lambda \in B\left[0, \hat{l}_{s}\right]}}\left|D_{1} \hat{L}_{r e s, n}(s, \lambda)\right|\left|s-s_{n}\right|+\sup _{\substack{s \in B\left[s_{n}, \rho_{n}\right] \\
\lambda \in B\left[0, \hat{l}_{s}\right]}}\left|D_{2} \hat{L}_{r e s, n}(s, \lambda)\right||\lambda| \\
& \leq \frac{\delta}{3}\left|s-s_{n}\right|+C|\lambda| .
\end{aligned}
$$


Hence $\left|T_{n}(s, \lambda)-s_{n}\right| \leq|\delta|^{-1}\left(\left|D_{2} \hat{L}\left(s_{n}, 0\right)\right||\lambda|+\frac{\delta}{3}\left|s-s_{n}\right|+C|\lambda|\right)$. Now we choose $\bar{l}_{s_{n}}$ such that for all $\lambda \in B\left[0, \bar{l}_{s_{n}}\right]$

$$
|\delta|^{-1}\left(\left|D_{2} \hat{L}\left(s_{n}, 0\right)\right||\lambda|+C|\lambda|\right)<\frac{1}{3} \rho_{n} .
$$

Then the lemma is true for $l_{s_{n}}:=\min \left\{\tilde{l}_{s_{n}}, \bar{l}_{s_{n}}\right\}$.

\section{Remark.}

(i) From $(17),(18)$ or (20) one finds that $\tilde{l}_{s_{n}}$ or $\bar{l}_{s_{n}}$ can be chosen such that

$$
\tilde{l}_{s_{n}}=\mathcal{O}(1 / n), \quad \text { or } \quad \bar{l}_{s_{n}}=\mathcal{O}\left(\rho_{n}\right)=\mathcal{O}\left(s_{n}\right)=\mathcal{O}\left(e^{-n}\right),
$$

respectively. Therefore $\bar{l}_{s_{n}}<\tilde{l}_{s_{n}}$ for sufficiently large $n$.

Furthermore, because of (19) there is a $K$ such that $D_{2} \hat{L}\left(s_{n}, 0\right)<K$ for all sufficiently large $n$. Therefore $\delta^{-1}(K+C)|\lambda|<(1 / 3) \rho_{n}$ implies (20). Altogether, for sufficiently large $n$ we can choose

$$
l_{s_{n}}=\frac{\delta}{3}(K+C)^{-1} \rho_{n} .
$$

(ii) The quantities $\bar{\beta}$ and $\tilde{l}_{s_{n}}$ can be chosen in such a way that in addition to (18) also $\left|D_{2} \hat{L}_{r e s, n}\left(s_{n} \beta, \lambda\right)\right|<\frac{\delta}{3}$ for all $\beta \in[1-\bar{\beta}, 1+\bar{\beta}]$ and all $\lambda \in B\left[0, \tilde{l}_{s_{n}}\right]$.

Now we return to the original fixed point equation (15). First we introduce some simplifying notations.

Let $\mathbf{r}:=\left(r_{1}^{1}, \ldots, r_{1}^{\left\lfloor\frac{N}{2}\right\rfloor}, r_{2}^{2}, \ldots, r_{2}^{\left\lfloor\frac{N+1}{2}\right\rfloor}\right) \in \mathbb{R}_{+}^{N-1}$. We define projections $\pi_{k}^{i}$ as follows

$$
\pi_{k}^{i}: \mathbb{R}_{+}^{N-1} \rightarrow \mathbb{R}_{+}, \quad \pi_{k}^{i}\left(\left(r_{1}^{1}, \ldots, r_{1}^{\left\lfloor\frac{N}{2}\right\rfloor}, r_{2}^{2}, \ldots, r_{2}^{\left\lfloor\frac{N+1}{2}\right\rfloor}\right)\right)=r_{k}^{i}
$$

Now let $\hat{\mathbf{L}}(\hat{\mathbf{r}}, 0)=0$. By Lemma 4.6, each $\hat{r}_{k}^{i}$ has a $\rho_{k}^{i}=\hat{r}_{k}^{i} \bar{\beta}_{k}$ and a corresponding $B_{k}^{i}:=B\left[\hat{r}_{k}^{i}, \rho_{k}^{i}\right]$ assigned to it. With these we define the cylinder

$$
\mathfrak{C}_{\hat{\mathbf{r}}}:=B_{1}^{1} \times \ldots \times B_{1}^{\left\lfloor\frac{N}{2}\right\rfloor} \times B_{2}^{2} \times \ldots \times B_{2}^{\left\lfloor\frac{N+1}{2}\right\rfloor} .
$$

Further we define

$$
\bar{\beta}:=\max \left\{\bar{\beta}_{1}, \bar{\beta}_{2}\right\}, \quad \underline{\beta}:=\min \left\{\bar{\beta}_{1}, \bar{\beta}_{2}\right\}
$$

and similarly

$$
\bar{\rho}(\hat{\mathbf{r}}):=\max \left\{\rho_{k}^{i}\right\}, \quad \underline{\rho}(\hat{\mathbf{r}}):=\min \left\{\rho_{k}^{i}\right\} .
$$


For $\mathbf{r}=\left(r^{1}, \ldots, r^{N-1}\right) \in \mathbb{R}_{+}^{N-1} \subset \mathbb{R}^{N-1}$ we introduce

$$
\overline{\mathbf{r}}:=\max \left\{r^{i}, i=1, \ldots, N-1\right\}=\|\mathbf{r}\|, \quad \underline{\mathbf{r}}:=\min \left\{r^{i}, i=1, \ldots, N-1\right\},
$$

and with that we define

$$
\kappa(\mathbf{r}):=\underline{\mathbf{r}} / \overline{\mathbf{r}}=\underline{\mathbf{r}} /\|\mathbf{r}\|
$$

From these definitions it follows

$$
\underline{\hat{\mathbf{r}}} \beta \leq \underline{\rho}(\hat{\mathbf{r}}) \leq \bar{\rho}(\hat{\mathbf{r}}) \leq\|\hat{\mathbf{r}}\| \bar{\beta}
$$

Therefore $\sup \left\{\|\mathbf{r}\|: \mathbf{r} \in \mathfrak{C}_{\hat{\mathbf{r}}}\right\} \leq\|\hat{\mathbf{r}}\|(1+\bar{\beta})$.

In accordance with $(21)$ there is a constant $\hat{K}$ such that $l_{\underline{\underline{\hat{r}}}}=\hat{K} \underline{\rho}(\hat{\mathbf{r}})$. Finally we define $\mathfrak{B}_{\hat{\mathbf{r}}}:=B\left[0, l_{\hat{\mathbf{r}}}\right]$.

Lemma 4.7 Let $\kappa^{*} \in(0,1]$. There is an $\eta=\eta\left(\kappa^{*}\right)$ such that for all $\hat{\mathbf{r}}$ with $\kappa(\hat{\mathbf{r}}) \geq \kappa^{*},\|\hat{\mathbf{r}}\|<\eta$ there is an $\epsilon_{r}$ such that for all $r \in\left(0, \epsilon_{r}\right)$ the fixed point equation (15) has a unique fixed point $\left(\mathbf{r}_{\hat{\mathbf{r}}}, \lambda_{\hat{\mathbf{r}}}\right)(r) \in \mathfrak{C}_{\hat{\mathbf{r}}} \times \mathfrak{B}_{\hat{\mathbf{r}}}$. Moreover, $\mathbf{r}_{\hat{\mathbf{r}}}$ and $\lambda_{\hat{\mathbf{r}}}$ depend smoothly on $r$.

Proof. First we show that there is an appropriate $\tilde{\eta}$ such that $\mathcal{T}_{\hat{\mathbf{r}}}(r, \cdot, \cdot)$ is a contraction on $\mathfrak{C}_{\hat{\mathbf{r}}} \times \mathfrak{B}_{\hat{\mathbf{r}}}$. We introduce for $(\mathbf{r}, \lambda) \in \mathbb{R}^{N-1} \times \mathbb{R}$ the norm $\|(\mathbf{r}, \lambda)\|:=\|\mathbf{r}\|+|\lambda|$. Then, from the definition of $\mathcal{T}_{\hat{\mathbf{r}}}$, see (15), we find:

$$
\begin{aligned}
\left\|D_{(2,3)} \mathcal{T}_{\hat{\mathbf{r}}}(r, \mathbf{r}, \lambda)\right\| \leq & \left\|\mathcal{D}^{-1} D_{2} \hat{\mathbf{L}}(\hat{\mathbf{r}}, 0)\right\| \\
& +\max \left\{\left\|\mathcal{D}^{-1} D_{1} \hat{\mathbf{L}}_{r e s, \hat{\mathbf{r}}}(\mathbf{r}, \lambda)\right\|,\left\|\mathcal{D}^{-1} D_{2} \hat{\mathbf{L}}_{r e s, \hat{\mathbf{r}}}(\mathbf{r}, \lambda)\right\|\right\} \\
& +\left\|(\mathcal{M} \hat{\mathcal{D}})^{-1}\right\|\left\|D_{(2,3)} \hat{\mathcal{R}}(r, \mathbf{r}, \lambda)\right\| .
\end{aligned}
$$

Because of (19) there is an $\eta_{1}$ such that $\|\hat{\mathbf{r}}\|<\eta_{1} / 1+\bar{\beta}$ implies

$$
\left\|\mathcal{D}^{-1} D_{2} \hat{\mathbf{L}}(\hat{\mathbf{r}}, 0)\right\|<\frac{1}{3}
$$

Moreover, according to Lemma 4.6 and the remark following its proof the $\eta_{1}$ can be chosen such that for $\|\hat{\mathbf{r}}\|<\eta_{1} / 1+\bar{\beta}$ and $(\mathbf{r}, \lambda) \in \mathfrak{C}_{\hat{\mathbf{r}}} \times \mathfrak{B}_{\hat{\mathbf{r}}}$ also

$$
\left\|\mathcal{D}^{-1} D_{1} \hat{\mathbf{L}}_{r e s, \hat{\mathbf{r}}}(\mathbf{r}, \lambda)\right\|<\frac{1}{3}, \quad\left\|\mathcal{D}^{-1} D_{2} \hat{\mathbf{L}}_{r e s, \hat{\mathbf{r}}}(\mathbf{r}, \lambda)\right\|<\frac{1}{3} .
$$

According to Corollary 4.5 there are an $\tilde{\epsilon}_{r}$ and an $\eta_{2}<\eta_{1}$ such that for all $r<\tilde{\epsilon}_{r}$, all $\mathbf{r}$ with $\|\mathbf{r}\|<\eta_{2}$ and all $\lambda \in \mathcal{B}_{\hat{\mathbf{r}}}$ we have

$$
\left\|(\mathcal{M D})^{-1}\right\|\left\|D_{(2,3)} \hat{\mathcal{R}}(r, \mathbf{r}, \lambda)\right\|<\frac{1}{6} .
$$


Therefore, if $\|\hat{\mathbf{r}}\|(1+\bar{\beta})<\eta_{2}$, then (23) holds true for all $\mathbf{r} \in \mathfrak{C}_{\hat{\mathbf{r}}}$.

Thus, we have shown that if $\|\hat{\mathbf{r}}\|<\eta_{2} / 1+\bar{\beta}$ then $\left\|D_{(2,3)} \mathcal{T}_{\hat{\mathbf{r}}}(r, \mathbf{r}, \lambda)\right\|<1$ for all $(\mathbf{r}, \lambda) \in \mathfrak{C}_{\hat{\mathbf{r}}} \times \mathfrak{B}_{\hat{\mathbf{r}}}, r<\tilde{\epsilon}_{r}$. In other words, the mapping $\mathcal{T}_{\hat{\mathbf{r}}}(r, \cdot, \cdot)$ is a contraction on $\mathfrak{C}_{\hat{\mathbf{r}}} \times \mathfrak{B}_{\hat{\mathbf{r}}}$.

Next we verify that $\mathcal{T}_{\hat{\mathbf{r}}}(r, \cdot, \cdot)$ maps $\mathfrak{C}_{\hat{\mathbf{r}}} \times \mathfrak{B}_{\hat{\mathbf{r}}}$ into itself. Lemma 4.6 provides that for all $(\mathbf{r}, \lambda) \in \mathfrak{C}_{\hat{\mathbf{r}}} \times \mathfrak{B}_{\hat{\mathbf{r}}}$

$$
\left|\pi_{k}^{i}\left(\hat{\mathbf{r}}-\mathcal{D}^{-1}\left(D_{2} \hat{\mathbf{L}}(\hat{\mathbf{r}}, 0) \lambda+\hat{\mathbf{L}}_{r e s, \hat{\mathbf{r}}}(\mathbf{r}, \lambda)\right)\right)\right|<\frac{2}{3} \rho_{k}^{i} .
$$

It remains, see $(15)$, to consider the term $(\mathcal{M} \hat{\mathcal{D}})^{-1} \hat{\mathcal{R}}(r, \mathbf{r}, \lambda)$. Let $(\mathbf{r}, \lambda) \in$ $\mathfrak{C}_{\hat{\mathbf{r}}} \times \mathfrak{B}_{\hat{\mathbf{r}}}$. In accordance with Corollary 4.4 there are constants $C$ and $\hat{C}$ such that

$$
\|\hat{\mathcal{R}}(r, \mathbf{r}, \lambda)\| \leq C r+\hat{C}\|\hat{\mathbf{r}}\|^{\alpha} .
$$

For any given $\hat{\mathbf{r}}$ we can choose $\epsilon_{r}=\epsilon_{r}(\hat{\mathbf{r}})<\tilde{\epsilon}_{r}$ that small such that

$$
\left\|(\mathcal{M} \hat{\mathcal{D}})^{-1}\right\| C r<\min \left\{(1 / 6) \underline{\rho}(\hat{\mathbf{r}}),(1 / 2) l_{\underline{\hat{\hat{}}}}\right\} .
$$

Further, due to $(22)$ we find that $\underline{\rho}(\hat{\mathbf{r}}) \geq \underline{\beta} \kappa^{*}\|\hat{\mathbf{r}}\|$, and because of $l_{\underline{\mathbf{r}}}=\hat{K} \underline{\rho}(\hat{\mathbf{r}})$ we have $l_{\hat{\mathbf{r}}} \geq \hat{K} \underline{\beta} \kappa^{*}\|\hat{\mathbf{r}}\|$. Hence there is a $\eta_{3} \leq \eta_{2}$ such that for $\hat{\mathbf{r}},\|\hat{\mathbf{r}}\|(1+\bar{\beta})<\eta_{3}$

$$
\left\|(\mathcal{M \mathcal { D }})^{-1}\right\| \hat{C}\|\hat{\mathbf{r}}\|^{\alpha}<\min \left\{(1 / 6) \underline{\rho}(\hat{\mathbf{r}}),(1 / 2) l_{\underline{\hat{r}}}\right\} .
$$

This finally shows for those $\hat{\mathbf{r}}$ and $r<\epsilon_{r}$

$$
\left\|(\mathcal{M} \hat{\mathcal{D}})^{-1}\right\|\|\hat{\mathcal{R}}(r, \mathbf{r}, \lambda)\| \leq \min \left\{(1 / 3) \underline{\rho}(\hat{\mathbf{r}}), l_{\hat{\underline{r}}}\right\} .
$$

Together with (24) this implies that $\mathcal{T}_{\hat{\mathbf{r}}}(r, \cdot, \cdot)$ maps $\mathfrak{C}_{\hat{\mathbf{r}}} \times \mathfrak{B}_{\hat{\mathbf{r}}}$ into itself.

Now we can apply the Banach Fixed Point Theorem to prove the existence of $\left(\mathbf{r}_{\hat{\mathbf{r}}}, \lambda_{\hat{\mathbf{r}}}\right)(r)$.

Finally, let $(\mathbf{r}, \lambda)=\mathcal{T}_{\hat{\mathbf{r}}}(r, \mathbf{r}, \lambda)$. Applying the Implicit Function Theorem provides the smooth dependence of $\left(\mathbf{r}_{\hat{\mathbf{r}}}, \lambda_{\hat{\mathbf{r}}}\right)$ on $r$.

To complete the proof of Theorem 3.1 we consider the function $\lambda_{\hat{\mathbf{r}}}$. Our above considerations show that in particular

$$
\hat{\Xi}^{1}\left(r, \mathbf{r}_{\hat{\mathbf{r}}}(r), \lambda_{\hat{\mathbf{r}}}(r)\right)=\lambda_{\hat{\mathbf{r}}}(r)+\hat{L}_{2}\left(r, \lambda_{\hat{\mathbf{r}}}(r)\right)+\mathcal{O}\left(r^{\alpha}\right) \equiv 0 .
$$

Due to the structure of $\hat{L}_{2}$ the function $\lambda_{\hat{\mathbf{r}}}$ has infinitely many zeros. Moreover it follows that $\lim _{r \rightarrow 0} \lambda_{\hat{\mathbf{r}}}(r)=0$. Let $r_{0}$, such that $\lambda_{\hat{\mathbf{r}}}\left(r_{0}\right)=0$. Then $\left(r_{0}, \mathbf{r}_{\hat{\mathbf{r}}}\left(r_{0}\right)\right)$ corresponds to an $N$-homoclinic orbit $\mathcal{H}\left(r_{0}\right)$ (to $p_{1}$ ) of the vector field $f(\cdot, 0)$. 
Let $\lambda_{\mathcal{H}\left(r_{0}\right)}(\omega):=\lambda_{\hat{\mathbf{r}}}(r(\omega))$; defined on some interval $(a, \infty)$. Then $\lambda_{\mathcal{H}\left(r_{0}\right)}$ has infinitely many zeros, and $\left|\lambda_{\mathcal{H}\left(r_{0}\right)}(\omega)\right|$ tends exponentially fast to zero as $\omega$ tends to infinity.

\subsection{Further homoclinic orbits}

So far we have proved the existence of infinitely many $N$-homoclinic solutions to the equilibrium $p_{1}$, which lie on snaking curves and whose 'outer' pulses become wider when one continues the orbits on the snaking curves. Similarly, we can prove the existence of such orbits to the equilibrium $p_{2}$.

Let $\mathcal{H}\left(\lambda_{\hat{\mathbf{r}}}(r)\right)$ be the $N$-homoclinic orbits corresponding to the snaking curve $\left\{\left(r, \lambda_{\hat{\mathbf{r}}}(r)\right)\right\}$. Then, in the sense of the Hausdorff metric, the following limits exists

$$
\lim _{r \rightarrow 0} \mathcal{H}\left(\lambda_{\hat{\mathbf{r}}}(r)\right)=\Gamma \cup \mathcal{H}_{2, N-1}(\hat{\mathbf{r}}),
$$

where $\mathcal{H}_{2, N-1}(\hat{\mathbf{r}})$ is a particular (symmetric) $N$ - 1-homoclinic orbit to $p_{2}$. Roughly speaking, for $\omega_{2}^{1} \rightarrow \infty$ (or $r \rightarrow 0$, resp.) the system (11) decouples: $\Xi^{1}=\lambda=0$ models the break up of the original cycle $\Gamma$; and the solutions of $\Xi^{2}=0, \ldots, \Xi^{N}=0$ correspond to symmetric $N$-homoclinic orbits to $p_{2}$. We will call $\Gamma \cup \mathcal{H}_{2, N-1}(\hat{\mathbf{r}})$ the snaking limit of $\mathcal{H}\left(\lambda_{\hat{\mathbf{r}}}(r)\right)$.

The numerical experiments for the generalized Swift-Hohenberg equation, however, show that there are also snaking curves different from the ones we described so far analytically. In Figure 4 there are 3-homoclinic orbits depicted where the snaking is due to the middle part - in other words the snaking curve is parametrized by $\omega_{2}^{2}$. The snaking limit of those orbits is a (symmetric) 2heteroclinic cycle connecting $p_{1}$ and $p_{2}$. In what follows we will make this statement somewhat more rigorous.

In the sketch of our further analysis we restrict ourselves to the consideration of symmetric 3-homoclinic orbits and show that another behaviour along snaking curves can occur. However, the arguments we will utilize can be generalized to arbitrary $N$-homoclinic orbits, for which we expect to find $\lfloor(N+1) / 2\rfloor$ different types of behaviour along snaking curves.

First we make clear that near $\Gamma$ there are infinitely many 2-heteroclinic cycles 
connecting $p_{1}$ and $p_{2}$. The bifurcation equation for those orbits reads:

$$
\begin{aligned}
& 0=\hat{\Xi}^{1}=\lambda+\hat{L}_{2}\left(r_{2}^{1}, \lambda\right)+\hat{\mathcal{R}}_{0}^{1} \\
& 0=\hat{\Xi}^{2}=\lambda+\hat{L}_{1}\left(r_{1}^{1}, \lambda\right)+\hat{\mathcal{R}}_{0}^{2}, \\
& 0=\hat{\Xi}^{3}=\lambda+\hat{L}_{1}\left(r_{1}^{1}, \lambda\right)+\hat{L}_{2}\left(r_{2}^{1}, \lambda\right)+\hat{\mathcal{R}}_{0}^{3},
\end{aligned}
$$

here $\hat{\mathcal{R}}^{1}=\mathcal{O}\left(\left(r_{2}^{1}\right)^{\alpha}\right), \hat{\mathcal{R}}^{2}=\mathcal{O}\left(\left(r_{1}^{1}\right)^{\alpha}\right)$ and $\hat{\mathcal{R}}^{3}=\mathcal{O}\left(\left(r_{1}^{1}\right)^{\alpha}\right)+\mathcal{O}\left(\left(r_{2}^{1}\right)^{\alpha}\right)$; the lower index 0 should indicate that these $\hat{\mathcal{R}}$ do not depend on $r_{2}^{2}$. In the same way as outlined in Section 4.2 this system can be written as a fixed point equation

$$
\left(r_{1}^{1}, r_{2}^{1}, \lambda\right)=T_{\hat{\mathbf{r}}}\left(r_{1}^{1}, r_{2}^{1}, \lambda\right)
$$

Let $\mathbf{r}:=\left(r_{1}^{1}, r_{2}^{1}\right)$, and with that let $\hat{\mathbf{r}}$ have the same meaning as in Section 4.2. Using the arguments of the proof of Lemma 4.7 we find that (26) has an unique fixed point $\left(\mathbf{r}_{\hat{\mathbf{r}}}, \lambda_{\hat{\mathbf{r}}}\right)$ in $\mathfrak{C}_{\hat{\mathbf{r}}} \times\left[-\epsilon_{\hat{\mathbf{r}}}, \epsilon_{\hat{\mathbf{r}}}\right]$. Moreover, if the vector field $f$ is conservative, as in the example of Equation (1), then $\lambda_{\hat{\mathbf{r}}}=0$ for all $\hat{\mathbf{r}}$. (For $\lambda \neq 0$ the fixed points $p_{1}$ and $p_{2}$ are in different level sets - therefore they cannot be connected by a heteroclinic orbit.)

Now, each of these symmetric 2-heteroclinic cycles can be seen as a primary cycle in its own right. Then our above considerations show that there are onehomoclinic orbits (to $p_{1}$ ) having this cycle as snaking limit. But related to $\Gamma$ these orbits are the observed 3-homoclinic orbits.

More precisely, in accordance with Corollary 4.4 the bifurcation equation for 3-homoclinic orbits reads:

$$
\begin{aligned}
& 0=\hat{\Xi}^{1}=\lambda+\hat{L}_{2}\left(r_{2}^{1}, \lambda\right)+\hat{\mathcal{R}}^{1} \\
& 0=\hat{\Xi}^{2}=\lambda+\hat{L}_{1}\left(r_{1}^{1}, \lambda\right)+\hat{L}_{2}\left(r_{2}^{2}, \lambda\right)+\hat{\mathcal{R}}^{2}, \\
& 0=\hat{\Xi}^{3}=\lambda+\hat{L}_{1}\left(r_{1}^{1}, \lambda\right)+\hat{L}_{2}\left(r_{2}^{1}, \lambda\right)+\hat{\mathcal{R}}^{3} .
\end{aligned}
$$

In Section 4.3 we have solved this equation for $\left(r_{1}^{1}, r_{2}^{2}, \lambda\right)=\left(\bar{r}_{1}^{1}, \bar{r}_{2}^{2}, \bar{\lambda}\right)\left(r_{2}^{1}\right)$. The corresponding snaking curve is parametrized by $\omega_{2}^{1}$. In the same way (27) can be solved for $\left(r_{1}^{1}, r_{2}^{1}, \lambda\right)=\left(\bar{r}_{1}^{1}, \bar{r}_{2}^{1}, \overline{\bar{\lambda}}\right)\left(r_{2}^{2}\right)$. Then the corresponding snaking curve is parametrized by $\omega_{2}^{2}$.

Indeed, the right-hand side of (27) tends to the right-hand side of (25) as $r_{2}^{2}$ tends to zero. This shows also along this way that the snaking limit of $\mathcal{H}\left(\overline{\bar{\lambda}}\left(r_{2}^{2}\right)\right)$ is a 2-heteroclinic cycle. 
Our analysis shows in particular that for all $r_{2}^{1}$ and $r_{2}^{2}$

$$
\left(\bar{r}_{1}^{1}\left(r_{2}^{1}\right), r_{2}^{1}, \bar{r}_{2}^{2}\left(r_{2}^{1}\right), \bar{\lambda}\left(r_{2}^{1}\right)\right) \neq\left(\overline{\bar{r}}_{1}^{1}\left(r_{2}^{2}\right), \overline{\bar{r}}_{2}^{1}\left(r_{2}^{2}\right), r_{2}^{2}, \overline{\bar{\lambda}}\left(r_{2}^{2}\right)\right) .
$$

In other words, there is no symmetric 3-homoclinic orbit that snakes with respect to both $\omega_{2}^{1}$ and $\omega_{2}^{2}$. This means that for sufficiently large transition times there will be no fold bifurcations as in Figure 4, where the red and green branches meet. (The bifurcations in this Figure are outside the reference of the presented analysis, since the $r_{k}^{i}$ are too large.)

Finally, we note that only the times $\omega_{2}^{i}$ can serve as snaking parameters and not the $\omega_{1}^{i}$. This mean that the times separating pulses stay almost constant along snaking curves.

\section{Conclusions}

In this paper we have studied the emergence of $N$-homoclinic orbits near heteroclinic cycles between equilibria of saddle-focus type (EE cycles) in reversible systems. We have shown that for each $N$ there are infinitely many $N$-homoclinic orbits, which exist on (distinct) snaking curves near the cycle. We have focussed on the case where these curves are parameterized by a transition time $\omega_{2}^{1}$ and have discussed other possibilities briefly.

It is interesting to note that the behaviour of $N$-homoclinic orbits reveals a striking difference in the dynamics near EE cycles and EP cycles. Note first of all that EP cycles exist robustly in reversible systems, and therefore the fold points along snaking curves of homoclinic orbits in their neighbourhood do not converge to a single parameter value, but rather oscillate between 2 values, which are the boundaries of the parameter interval, in which the cycle exists, see also Figure 6.

This global character of bifurcations near an EP cycle makes a rigorous analytical treatment difficult. Nevertheless, numerical results for $N$-homoclinic orbits near such a cycle have been described in $[5,17]$

Below we present a corresponding result for the generalized Swift-Hohenberg Equation (1). Similar to Section 2 we view the equation as depending on $r$, this time setting $q_{c}=0.5, v=0.41$ and $g=1$. And as in that section, onehomoclinic orbits to 0 are computed using shooting, and two-homoclinic orbits by the branch-switching method.

In Figure 6 we present a bifurcation diagram for symmetric 2-homoclinic orbits to 0 near an EP cycle, which exists for $r \in[-0.0146,-0.0125]$. This EP cycle generates two snaking curves of one-homoclinic orbits, one which is shown 


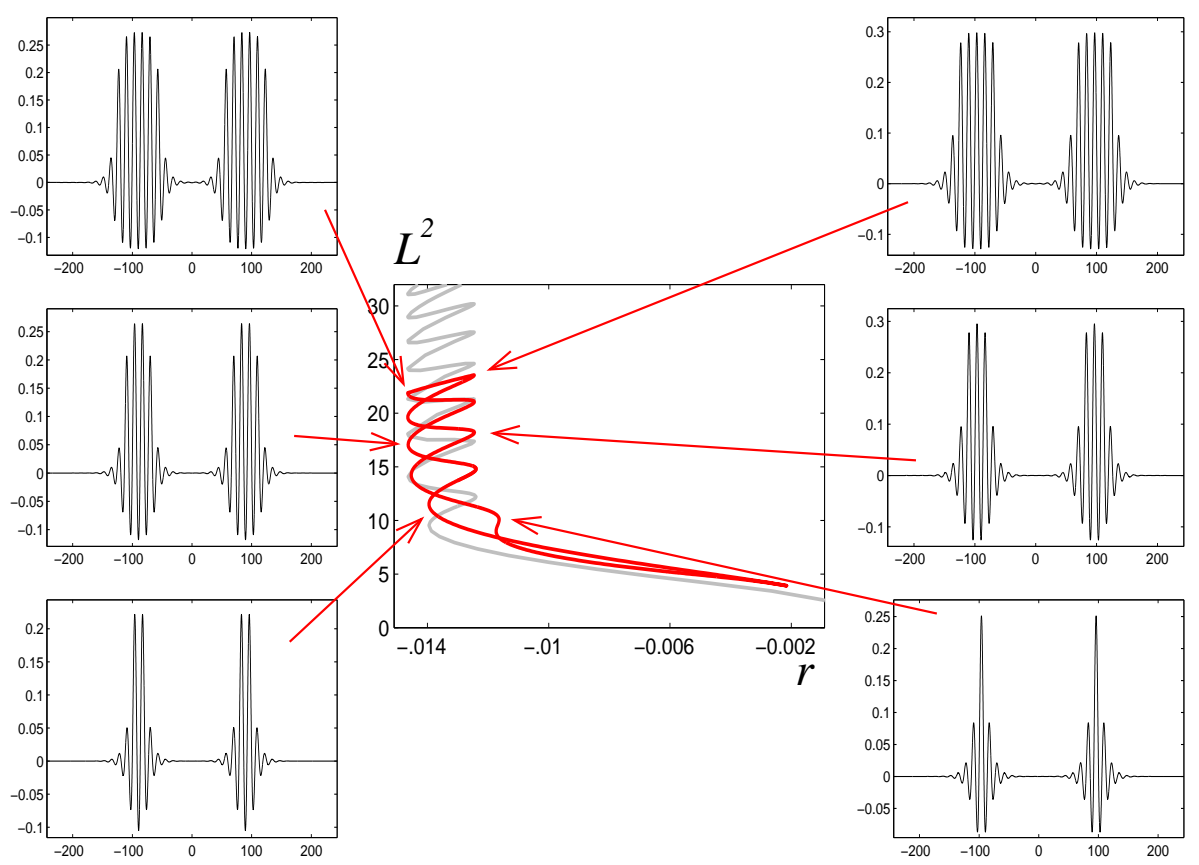

Figure 6. 2-homoclinic orbits near an EP cycle exist along an isola in (1).

in grey in the Figure. In addition, the rescaled continuation curve for a twohomoclinic orbit is shown in red in the Figure. And although this curve follows (parts of) the snaking curve, we see a clear difference. In contrast to the EE cycle case the 2-homoclinic orbit does not exist on a snaking curve, but rather on an isola in the diagram.

A reason for this becomes apparent when we consider the plots for the twohomoclinic solutions along the snaking curve. Moving up on the bifurcation curve we see that the pulses of the solutions become wider, but in contrast to the EE cycle case, the pulses grow symmetrically about their centre, such that they do not stay separated but approach each other. (Note that instead of approaching an equilibrium solution the pulses now come close to a periodic orbit such that they grow additional oscillations.). Hence, in the notation of Section 4 , the time $\omega_{1}^{1}$ decreases along the snaking curve. And this process cannot be repeated ad infinitum, since the 2 pulses would have to meet, and therefore the 2-homoclinic orbit cannot follow the full snaking curve. A similar behaviour has been found for different examples in $[5,17]$. Indeed, so far 2homoclinic orbits near EP cycles have only been found to lie on isolas and not snaking curves.

It is one goal of our future research to better understand the dynamics near EP cycles. For this we will extend Lin's method such that it can deal with cycles involving periodic orbits. The necessary foundations will be presented in a forthcoming paper. Besides, we want to understand the geometrical reason for the different behaviour of $N$-homoclinic orbits near EE cycles and EP cycles, respectively. 


\section{References}

[1] J. Burke and E. Knobloch. Localized states in the generalized Swift-Hohenberg equation. Phys. Rev. E, 73:056211, 2006

[2] M. Chen. Solitary-wave and multi-pulsed travelling-wave solutions of Boussinesq systems. Appl. Anal., 75(1-2):213-240, 2000.

[3] P. Coullet, C. Riera and C. Tresser. Stable Static Localized Structures in One Dimension. Phys. Rev. Letters, 84(14):3069-3072, 2000.

[4] J. Härterich. Cascades of homoclinic orbits in reversible dynamical systems. Physica D, 112:187-200, 1998.

[5] G. H. M. van der Heijden, A. R. Champneys and J. M. T. Thompson. Spatially complex localisation in twisted elastic rods constrained to a cylinder. International Journal of Solids and Structures, 39:1863-1883, 2002.

[6] M. F. Hilali, S. Métens, P. Borckmans and G. Dewel. Pattern selection in the generalized Swift-Hohenberg model. Phys. Rev. E, 51(3):2046-2052, 1995.

[7] G. W. Hunt, M. A. Peletier, A. R. Champneys, P. D. Woods, M. Ahmer Wadee, C. J. Budd and G. J. Lord. Cellular buckling in long structures. Nonlinear Dynamics, 21:3-29, 2000.

[8] J. Knobloch. Lin's method for discrete and continuous dynamical systems and applications. TU Ilmenau 2004. http://imath.mathematik.tu-ilmenau.de/ knobi

[9] J. Knobloch, J. S. W. Lamb and K. Webster, Shift dynamics in a neighborhood of a T-point containing two saddle-foci. In preparation.

[10] J. Knobloch and T. Wagenknecht. Homoclinic snaking near a heteroclinic cycle in reversible systems. Physica D, 206:82-93, 2005.

[11] J. S. W. Lamb and J. A. G Roberts. Time-reversal symmetry in dynamical systems: A survey. Physica D, 112:1-39, 1998.

[12] B. E. Oldeman, A. R. Champneys and B. Krauskopf Homoclinic branch switching: a numerical implementation of Lin's method Int. Journal of Bifurcation and Chaos, 13:2977-3000, 2003.

[13] B. Sandstede. Verzweigungstheorie homokliner Verdopplungen. IAAS Report No. 7, 1993.

[14] B. Sandstede. Instability of localised buckling modes in a one-dimensional strut model. Phil. Trans. Roy. Soc. Lond. A, 355:2083-2097, 1997.

[15] M. Tlidi, P. Mandel and R. Lefever. Localized Structures and Localized Patterns in Optical Bistability. Phys. Rev. Letters, 73(5):640-643, 1994.

[16] A. Vanderbauwhede and B. Fiedler. Homoclinic period blow-up in reversible and conservative systems. Z. Angew. Math. Phys., 43:292-318, 1992. 
[17] M. K. Wadee, C. D. Coman and A. P. Bassom. Solitary wave interaction phenomena in a strut buckling model incorporating restabilisation. Physica D, 163:26-48, 2002.

[18] P. D. Woods and A. R. Champneys. Heteroclinic tangles and homoclinic snaking in the unfolding of a degenerate reversible Hamiltonian Hopf bifurcation. Physica $D, 129: 147-170$.

[19] E. J. Doedel, A. R. Champneys, T. R. Fairgrieve, Yu. A. Kuznetsov, B. Sandstede, and X. J. Wang. AUTO97 continuation and bifurcation software for ordinary differential equations, 1997. Available by anonymous ftp from FTP.CS.CONCORDIA.CA, directory PUB/DOEDEL/AUTO. 